\title{
TESTES DE VIGOR PARA AVALIAÇÃO DO POTENCIAL FISIOLÓGICO DE SEMENTES DE MAMONA (Ricinus communis L.) ${ }^{1}$
}

\author{
Vigor test for the evaluation of the physiology potencial of castor bean (Ricinus communis L.) seeds
}

\author{
Rita de Cássia Mendes², Denise Cunha Fernandes dos Santos Dias³, \\ Marcio Dias Pereira ${ }^{4}$, Luiz Antonio dos Santos Dias ${ }^{3}$
}

\begin{abstract}
RESUMO
A qualidade de sementes de mamona tem sido avaliada rotineiramente pelo teste de germinação, cujos resultados, muitas vezes, não são confirmados em campo. Nesse contexto, os testes de vigor são essenciais, pois retratam o comportamento das sementes sob maior amplitude de ambiente. Neste trabalho, objetivou-se avaliar a eficiência de métodos para determinar o potencial fisiológico de sementes de mamona. Para tanto, foram utilizados sete lotes de sementes de mamona, cultivar AL-Guarany, apresentando teor de água de aproximadamente $7 \%$, que foram submetidos aos seguintes testes: germinação, primeira contagem de germinação, frio (a $10^{\circ} \mathrm{C} / 7$ dias e a $25^{\circ} \mathrm{C} / 5$ dias), porcentagem e velocidade de emergência de plântulas, envelhecimento acelerado a $41^{\circ} \mathrm{C}$ e $45^{\circ} \mathrm{C}$ e $100 \%$ UR, por 48, 72 e 96 horas e condutividade elétrica $\left(25\right.$ sementes embebidas em 75 e $100 \mathrm{~mL}$ de água destilada, a $25^{\circ} \mathrm{C}$, por $2,4,6,8$, 24,48 e 72 horas). Os testes de frio e de envelhecimento acelerado $\left(41^{\circ} \mathrm{C} / 72\right.$ horas e $100 \%$ UR) foram eficientes para avaliação do potencial fisiológico de sementes de mamona, permitindo classificação de lotes quanto ao vigor semelhante à emergência de plântulas em solo. O teste de condutividade elétrica não se mostrou adequado para avaliação do potencial fisiológico das sementes de mamona.
\end{abstract}

Termos para indexação: Vigor, germinação, envelhecimento acelerado, condutividade elétrica.

\begin{abstract}
This work had the objective to determine the efficiency of different tests for the evaluation of physiological potential of castor bean seeds. Seven seed lots of AL-Guarany cultivar were submitted to the following tests: seed moisture content, standard germination, first count, cold test $\left(10^{\circ} \mathrm{C} / 7\right.$ days followed by germination at $25^{\circ} \mathrm{C} / 5$ days), seedling emergence, speed emergence index, accelerated aging (at $41^{\circ} \mathrm{C}$ and $45^{\circ} \mathrm{C}$ and $100 \% \mathrm{RH}$, for 48,72 , and $96 \mathrm{~h}$ ) and electrical conductivity ( 25 seeds soaked into 75 and $100 \mathrm{~mL}$ of distilled water, at $25^{\circ} \mathrm{C}$, for $2,4,6,8,24,48$, and $72 \mathrm{~h}$ ). The trial was conducted in randomized completely design, with four replications. The means of treatments were compared by Tukey test at 5\% probability. Results showed that cold test and accelerated aging $\left(41^{\circ} \mathrm{C} / 72\right.$ hours) were efficient to evaluate the physiological potential of castor bean seeds, allowing the classification of lots regarding vigor to be similar to the seedling emergence test. Electrical conductivity test was not adequate for the evaluation of physiological potential of these seeds.
\end{abstract}

Index term: Vigor, germination, accelerated aging, electrical conductivity.

(Recebido em 4 de julho de 2008 e aprovado em 27 de novembro de 2008)

\section{INTRODUÇÃO}

O Brasil é um dos três maiores produtores mundiais de mamona (Ricinus communis L.), sendo o segundo exportador mundial de óleo obtido das sementes dessa oleaginosa (Savy Filho, 2005).

Dentre as oleaginosas promissoras para a produção de biodiesel, a mamona destaca-se como opção interessante para as regiões semiáridas, em razão de sua capacidade de produzir razoavelmente bem, sob condições de baixa precipitação pluvial, sendo considerada importante alternativa de trabalho e renda para os agricultores dessas regiões. Além disso, seu óleo é mais denso e viscoso que o proveniente de outras plantas como o dendê, girassol e algodão, sendo ainda o único na natureza solúvel em álcool, bastante versátil em termos de aplicação, podendo ser utilizado na indústria de plásticos, resinas, esmaltes, fibras, cosméticos e fármacos (Freire, 2001).

A utilização de sementes de alta qualidade constituise na base para a obtenção de estandes uniformes, plantas bem desenvolvidas, alta produtividade e elevado rendimento de óleo. Das sementes de mamona utilizadas em Minas Gerais no ano de 2007, 75\% apresentaram germinação inferior ao padrão estabelecido como mínimo para a comercialização dessas sementes no país que é de $80 \%$ de germinação (Departamento Federal de Agricultura de São Paulo-DFASP, 2007; Parrella et al., 2007).

\footnotetext{
${ }^{1}$ Parte da Dissertação apresentada à Coordenação do Curso de Pós-Graduação em Fitotecnia (Produção Vegetal) da UFV, defendida em 04/12/2007, como parte dos requisitos para obtenção do título de Magister Science

${ }^{2}$ Universidade Federal de Viçosa/UFV - Departamento de Fitotecnia Viçosa, MG

${ }^{3}$ Universidade Federal de Viçosa/UFV - Departamento de Fitotecnia - 36570-000 - Viçosa, MG - dcdias@ufv.br

${ }^{4}$ Universidade Federal de Viçosa/UFV - Departamento de Fitotecnia - Viçosa, MG
} 
A qualidade das sementes de mamona tem sido avaliada, rotineiramente, pelo teste de germinação (Brasil, 1992), conduzido sob condições ideais, em laboratório. Desse modo, os resultados obtidos expressam o potencial máximo de germinação do lote. No entanto, estes resultados nem sempre são confirmados em campo, principalmente quando as condições de ambiente se desviam das mais adequadas. Por este motivo, atualmente, é cada vez mais crescente a procura por métodos que permitam avaliar, de maneira rápida e eficiente, o potencial fisiológico das sementes, representado pelo seu potencial de armazenamento e de emergência de plântulas em campo. Para isso, destacam-se os testes de envelhecimento acelerado e condutividade elétrica que têm sido utilizados para sementes de diversas espécies. Porém, são escassos os estudos direcionados à adequação da metodologia desses testes para a avaliação do vigor de sementes de mamona.

O teste de envelhecimento acelerado baseia-se no princípio de que, quando as sementes são expostas, por determinado período, à alta temperatura e à alta umidade relativa do ar, o processo de deterioração é acelerado, de modo que sementes mais vigorosas deterioram-se mais lentamente (Marcos Filho, 1999). A combinação entre período de exposição e temperatura durante o teste varia de acordo com a espécie, havendo indicações de sucesso com o uso de temperaturas entre 40 e $45^{\circ} \mathrm{C}$ e períodos de envelhecimento de 48 a 96 horas para a maioria das culturas (Marcos Filho, 1999).

O teste de condutividade elétrica baseia-se na avaliação indireta do estado de organização das membranas celulares, por meio da determinação da quantidade de lixiviados liberados pelas sementes na solução de embebição (Vieira \& Krzyzanowski, 1999). Assim, sementes de menor vigor apresentam maior valor de condutividade elétrica quando comparadas às mais vigorosas (Association of Official Seed Analysts-AOSA, 1983; Hampton \& Tekrony, 1995).

Diversos fatores podem afetar os resultados de condutividade elétrica, dentre eles, o teor de água das sementes (Vanzolini \& Nakagawa, 1999), período de embebição (Loeffler et al., 1988; Dias \& Marcos Filho, 1996; Vanzolini \& Nakagawa, 1999), temperatura de embebição (Gaspar \& Nakagawa, 2002), volume de água utilizado e presença de danos nas sementes (Tao, 1978). Uma das vantagens desse teste é a rapidez na obtenção dos resultados (Vieira \& Krzyzanowski, 1999). O período de 24 horas de embebição tem sido o mais recomendado para a avaliação da condutividade elétrica de sementes de diversas espécies.
O uso de testes de vigor em programas de controle de qualidade de sementes é uma realidade para a maioria das grandes culturas e hortaliças de importância econômica. Porém, existem poucos relatos sobre a utilização desses testes em sementes de mamona.

Nesse contexto, conduziu-se este trabalho, com o onjetivo de avaliar a eficiência de diferentes métodos para determinação do potencial fisiológico de sementes de mamona, visando a destacar procedimentos mais promissores para utilização em programas de controle de qualidade.

\section{MATERIAL E MÉTODOS}

O trabalho foi desenvolvido no Laboratório de Pesquisa em Sementes do Departamento de Fitotecnia da Universidade Federal de Viçosa, no período de setembro/ 2006 a maio/2007, sendo utilizados sete lotes de sementes de mamona (Ricinus communis L.), cultivar AL-Guarany provenientes da Coordenadoria de Assistência Técnica Integral (CATI-SP) com grau de umidade em torno de 7,0\%. As sementes de cada lote foram acondicionadas em sacos de papel e mantidas em ambiente de laboratório, a $20^{\circ} \mathrm{C}$, durante todo o período de realização dos testes. Para cada lote, foram conduzidos os seguintes testes e determinações:

Germinação: oito subamostras de 25 sementes foram distribuídas sobre duas folhas de papel toalha e cobertas por outra folha do mesmo papel, umedecido com volume de água destilada equivalente a 2,5 vezes o peso do papel seco. Confeccionaram-se rolos que foram mantidos em germinador a $25^{\circ} \mathrm{C}$. As avaliações foram feitas aos sete e 14 dias após a semeadura, sendo os resultados expressos pela média das porcentagens de plântulas normais (Brasil, 1992).

Primeira contagem de germinação: conduzido juntamente com o teste de germinação, registrando-se a porcentagem de plântulas normais obtidas aos sete dias após a instalação do teste. Os resultados foram expressos em porcentagem média de plântulas normais.

Envelhecimento acelerado: foi realizado adotandose o método do gerbox adaptado recomendado pela AOSA (1983) e relatado por Marcos Filho (1999). Cerca de 250 sementes foram distribuídas em camada única sobre bandeja de tela acoplada à caixa gerbox, contendo, ao fundo, $40 \mathrm{~mL}$ de água. Os gerbox foram tampados, de modo a se obter $100 \%$ UR em seu interior, e mantidos em BOD a $41^{\circ} \mathrm{C}$ e $45^{\circ} \mathrm{C}$, durante 48,72 e 96 horas. Após cada período, oito subamostras de 25 sementes foram submetidas ao teste de germinação, conforme descrito acima. Os resultados foram expressos em porcentagem média de plântulas normais obtidas aos sete dias. 
Teste de frio: as sementes, em oito subamostras de 25 , foram distribuídas em papel toalha, seguindo-se a metodologia descrita anteriormente para o teste de germinação. Os rolos foram colocados em sacos plásticos e mantidos em incubadora $\mathrm{BOD}$, a $10^{\circ} \mathrm{C}$, por sete dias (Loeffler et al., 1985). Em seguida, os rolos foram transferidos para um germinador a $25^{\circ} \mathrm{C}$, onde permaneceram por 5 dias. Os resultados foram expressos em porcentagem média de plântulas apresentando comprimento igual ou superior a $5 \mathrm{~cm}$.

Condutividade elétrica: foi conduzido pelo método de massa, de acordo com o procedimento proposto pelo Comitê de Vigor da International Seed Testing Association-ISTA (1995) e relatado por Vieira \& Krzyzanowski (1999). Utilizaram-se quatro subamostras de 25 sementes, pesadas com precisão de 0,001g. As amostras foram colocadas em copos plásticos, contendo 75 e $100 \mathrm{~mL}$ de água destilada e foram mantidas em incubadora $\mathrm{BOD}, \mathrm{a} 25^{\circ} \mathrm{C}$, durante $2,4,6,8,24,48$ e 72 horas. Após cada período, a condutividade elétrica das soluções foi determinada em condutivímetro Digimed DM31 e os resultados expressos em $\mu \mathrm{S} / \mathrm{cm} / \mathrm{g}$.

Porcentagem e velocidade de emergência das plântulas: a semeadura foi feita em caixas plásticas, contendo uma mistura de areia e terra (proporção de $1: 1)$ umedecida a $70 \%$ da capacidade de campo, utilizando-se quatro subamostras de 50 sementes. As caixas foram mantidas em casa de vegetação, cuja temperatura mínima foi de $10 \pm 7^{\circ} \mathrm{C}$ e a máxima, $35 \pm 10^{\circ} \mathrm{C}$. As irrigações foram feitas sempre que necessário. Foram feitas contagens diárias do número de plântulas emergidas, até a estabilização das contagens, para cálculo do índice de velocidade de emergência (IVE) e da velocidade de emergência das plântulas (dias), de acordo com Nakagawa (1999). Foi calculada também, aos 12 e 20 dias após a semeadura, a porcentagem de emergência de plântulas.

Procedimento estatístico: o delineamento experimental utilizado foi o inteiramente casualizado, com quatro repetições. Os dados obtidos foram submetidos à análise de variância. Para cada teste, as médias obtidas para cada lote foram comparadas pelo teste de Tukey a $5 \%$ de probabilidade. Foram calculados ainda os coeficientes de correlação simples de Pearson (r) entre os dados obtidos nos testes de vigor e os de emergência das plântulas; a significância dos valores de $r$ foi determinada pelo teste $\mathrm{t}$, a 1 e $5 \%$ de probabilidade. As análises foram processadas no programa estatístico SAS Institute (1989).

\section{RESULTADOS E DISCUSSÃO}

Na tabela 1, encontram-se os resultados dos testes de germinação e de vigor realizados em sete lotes de sementes de mamona. Todos os lotes apresentaram germinação acima do padrão nacional mínimo (80\%) estabelecido para comercialização de sementes de mamona no país. Pelo teste de germinação, destacaram-se os lotes 2,5 , e 7 , os quais foram superiores aos lotes 3 e 6 . Ao se avaliar a primeira contagem do teste de germinação, observa-se a superioridade das sementes do lote $1 \mathrm{em}$ relação às dos demais lotes, com pior desempenho para o lote 5 , que não diferiu significativamente do lote 6 . O lote 6 também esteve entre os de menor qualidade pelos resultados de germinação. No entanto, o lote 5 , que teve

Tabela 1 - Valores médios de germinação (GERM), primeira contagem de germinação (PCG), germinação no teste de frio (TF), emergência de plântulas aos 12 dias (EMERG-12 dias) e aos 20 dias (EMERG-20 dias), velocidade de emergência de plântulas (VE) e índice de velocidade de emergência obtidos para os sete lotes de sementes de mamona.

\begin{tabular}{cccccccc}
\hline LOTE & GERM $(\%)$ & PCG $(\%)$ & TF $(\%)$ & $\begin{array}{c}\text { EMERG }- \\
12 \text { dias }(\%)\end{array}$ & $\begin{array}{c}\text { EMERG - } \\
20 \text { dias }(\%)\end{array}$ & $\begin{array}{c}\text { VE } \\
(\text { dias })\end{array}$ & IVE \\
\hline 1 & $93 \mathrm{AB}$ & $90 \mathrm{~A}$ & $85 \mathrm{~B}$ & $91 \mathrm{AB}$ & $95 \mathrm{~A}$ & $10,45 \mathrm{~A}$ & $9,11 \mathrm{~A}$ \\
2 & $94 \mathrm{~A}$ & $74 \mathrm{BC}$ & $94 \mathrm{~A}$ & $96 \mathrm{~A}$ & $99 \mathrm{~A}$ & $10,59 \mathrm{~A}$ & $9,37 \mathrm{~A}$ \\
3 & $84 \mathrm{BC}$ & $80 \mathrm{~B}$ & $93 \mathrm{~A}$ & $95 \mathrm{AB}$ & $98 \mathrm{~A}$ & $10,56 \mathrm{~A}$ & $9,35 \mathrm{~A}$ \\
4 & $89 \mathrm{ABC}$ & $73 \mathrm{BC}$ & $79 \mathrm{~B}$ & $84 \mathrm{~B}$ & $91 \mathrm{AB}$ & $11,40 \mathrm{BC}$ & $7,98 \mathrm{BC}$ \\
5 & $97 \mathrm{~A}$ & $63 \mathrm{D}$ & $84 \mathrm{~B}$ & $72 \mathrm{C}$ & $81 \mathrm{~B}$ & $11,68 \mathrm{C}$ & $6,97 \mathrm{D}$ \\
6 & $81 \mathrm{C}$ & $68 \mathrm{CD}$ & $62 \mathrm{C}$ & $75 \mathrm{C}$ & $81 \mathrm{~B}$ & $11,58 \mathrm{C}$ & $7,03 \mathrm{CD}$ \\
7 & $96 \mathrm{~A}$ & $73 \mathrm{BC}$ & $79 \mathrm{~B}$ & $92 \mathrm{AB}$ & $95 \mathrm{~A}$ & $10,91 \mathrm{AB}$ & $8,75 \mathrm{AB}$ \\
\hline $\mathrm{CV}(\%)$ & 5,2 & 5,8 & 4,1 & 6,2 & 4,8 & 1,9 & 5,1 \\
\hline
\end{tabular}

Médias seguidas da mesma letra, na coluna, não diferem pelo teste de Tukey a 5\% de probabilidade. 
fracodesempenho na primeira contagem de germinação, apresentou elevada germinação (97\%), estando entre os lotes de alta qualidade fisiológica pelos resultados deste teste (Tabela 1).

Ainda na Tabela 1, pelos resultados do teste de frio verifica-se maior vigor para as sementes dos lotes 2 e 3, vigor intermediário para os lotes $1,4,5$ e 7, com menor vigor para as sementes do lote 6 . O pior desempenho para as sementes deste lote também foi confirmado pelos resultados de germinação e de primeira contagem. Comparando-se os resultados de primeira contagem de germinação com os do teste de frio, constata-se que não houve concordância entre os mesmos quanto à identificação dos lotes de maior vigor.

Ao se avaliar a porcentagem de emergência de plântulas aos 12 dias (Tabela 1), destacaram-se os lotes 1, 2, 3 e 7 como superiores aos lotes 5 e 6 , identificados como os de pior desempenho em campo. Resultados semelhantes foram obtidos nos testes de emergência de plântulas aos 20 dias, velocidade de emergência e índice de velocidade de emergência (Tabela 1). Observa-se que o lote 5 apresentou alta germinação em laboratório, destacando-se entre os melhores, desempenho este que não se confirmou em campo. Em sementes de eritrina, Guedes et al. (2009) verificaram que os testes conduzidos em campo foram mais eficientes para a separação dos lotes em níveis de vigor.

Na Tabela 2, encontram-se os resultados do teste de condutividade elétrica para os sete lotes de sementes de mamona. Quando se utilizou o volume de água de $75 \mathrm{~mL}$, menores valores de condutividade elétrica foram obtidos, já, a partir de duas horas de embebição para o lote 6, indicando melhor qualidade fisiológica em relação aos demais lotes, resultado este que foi mantido até 8 horas de embebição. É importante ressaltar que esse lote foi classificado entre os de pior desempenho tanto no tesse de germinação como nos testes de vigor relacionados na Tabela 1. Nas avaliações feitas a partir de 24 horas de embebição, destacou-se o lote 1 como o de menor vigor, resultado este que novamente não se mostra coerente com o desempenho apresentado por este lote nos demais testes de avaliação da qualidade (Tabela 1). Portanto, o teste de condutividade elétrica não refletiu a qualidade fisiológica dos lotes de sementes de mamona, não sendo possível a identificação segura dos lotes de maior e menor vigor. Esses resultados estão de acordo com os obtidos por Fonseca et al. (2004), que concluíram que, em razão da baixa permeabilidade do tegumento das sementes, o teste de condutividade elétrica não permitiu diferenciar a qualidade fisiológica dos lotes de mamona. Parrella et al. (2007) também chegaram à conclusão de que o teste de condutividade elétrica, assim como o de lixiviação de K+, não são os mais indicados para a separação de lotes de sementes de mamona em diferentes níveis de qualidade. Por outro lado, Souza et al. (2007) verificaram que a condutividade elétrica, avaliada após 6 horas de embebição, em amostras de 25 sementes imersas em $75 \mathrm{~mL}$ de água deionizada foi eficiente para detectar diferenças de qualidade entre lotes de sementes de mamona. De acordo com Albuquerque et al. (2001), essa variação de resultados pode ser explicada uma vez que os testes podem ser afetados por características de composição química da cultivar, espessura do tegumento, tamanho das sementes, entre outros fatores.

Os resultados do teste de condutividade elétrica conduzido com $100 \mathrm{~mL}$ de água (Tabela 2) também não se mostraram coerentes com os demais testes de vigor empregados (Tabela 1) quanto à identificação dos lotes de melhor e pior desempenho, mantendo tendência de classificação de lotes semelhante à obtida no teste realizado com $75 \mathrm{~mL}$ de água (Tabela 2).

$\mathrm{Na}$ Tabela 3, são apresentados os resultados do teste de envelhecimento acelerado, nas diferentes temperaturas e períodos de exposição. Na temperatura de $41^{\circ} \mathrm{C}$ e período de exposição de 48 horas, o lote 1 , que apresentou alta qualidade nos demais testes de vigor (Tabela 1), foi classificado entre os lotes de pior desempenho, só não sendo inferior ao lote 6. Os lotes 2 e 3 apresentaram vigor superior aos demais. Quando se utilizou o período de 72 horas de envelhecimento, os lotes 1,2 e 3 foram considerados os de maior qualidade fisiológica e o lote 6 como o de pior vigor, resultados esses que se mostram coerentes com os obtidos nos demais testes de vigor aplicados (Tabela 1). A $41^{\circ} \mathrm{C}$ e 96 horas, manteve-se a mesma tendência de classificação de lotes obtida no período de 72 horas, porém, constatouse alta incidência de fungos, dificultando a avaliação, especialmente dos lotes de menor qualidade fisiológica. Dessa forma, os resultados obtidos para o teste de envelhecimento acelerado, a $41^{\circ} \mathrm{C}$ e $100 \%$ UR, indicaram que o período de 72 horas de envelhecimento permitiu a separação dos lotes em diferentes níveis de vigor, correspondendo à classificação de lotes obtidos nos demais testes empregados. Portanto, esse período mostrou-se mais adequado para a condução do teste que os demais tempos testados. Por sua vez, Rocha et al. (2007) constataram que o teste de envelhecimento acelerado a $40^{\circ} \mathrm{C}$ e $100 \%$ UR, por um período de exposição de 72 horas, provocou a morte das sementes de mamona BRS-Energia, o que não ocorreu quando foi utilizada umidade relativa de $76 \%$. 
Tabela 2 - Valores médios da condutividade elétrica $\left(\mu \mathrm{S} . \mathrm{cm}^{-1} \cdot \mathrm{g}^{-1}\right)$ de sete lotes de sementes de mamona imersos em 75 e 100mL de água, após diferentes períodos de embebição.

\begin{tabular}{|c|c|c|c|c|c|c|c|}
\hline \multirow[b]{3}{*}{ Lotes } & \multicolumn{7}{|c|}{ Volume de água $(75 \mathrm{ml})$} \\
\hline & \multicolumn{7}{|c|}{ Período de embebição (Horas) } \\
\hline & 2 & 4 & 6 & 8 & 24 & 48 & 72 \\
\hline 1 & $23,0 \mathrm{~A}$ & $26,1 \mathrm{~A}$ & $28,4 \mathrm{~A}$ & $30,8 \mathrm{~A}$ & $51,2 \mathrm{~A}$ & $71,8 \mathrm{~A}$ & $96,5 \mathrm{~A}$ \\
\hline 2 & $22,3 \mathrm{~A}$ & $24,8 \mathrm{~A}$ & $26,4 \mathrm{~A}$ & $27,9 \mathrm{~A}$ & $42,8 \mathrm{BC}$ & $56,2 \mathrm{~B}$ & $64,0 \mathrm{~B}$ \\
\hline 3 & $22,6 \mathrm{~A}$ & $25,1 \mathrm{~A}$ & $26,7 \mathrm{~A}$ & $28,2 \mathrm{~A}$ & $41,3 \mathrm{C}$ & $56,8 \mathrm{~B}$ & $65,7 \mathrm{~B}$ \\
\hline 4 & $21,3 \mathrm{~A}$ & $24,1 \mathrm{~A}$ & $26,5 \mathrm{~A}$ & $28,6 \mathrm{~A}$ & $44,9 \mathrm{BC}$ & $57,0 \mathrm{~B}$ & $64,1 \mathrm{~B}$ \\
\hline 5 & $21,6 \mathrm{~A}$ & $24,4 \mathrm{~A}$ & $26,7 \mathrm{~A}$ & $29,2 \mathrm{~A}$ & $43,6 \mathrm{BC}$ & $56,4 \mathrm{~B}$ & $67,7 \mathrm{~B}$ \\
\hline 6 & $17,6 \mathrm{~B}$ & 20,7 B & $22,9 \mathrm{~B}$ & $24,9 \mathrm{~B}$ & $41,8 \mathrm{BC}$ & $51,1 \mathrm{~B}$ & $59,3 \mathrm{~B}$ \\
\hline 7 & $22,7 \mathrm{~A}$ & $25,1 \mathrm{~A}$ & $27,2 \mathrm{~A}$ & $29,5 \mathrm{~A}$ & $46,2 \mathrm{~B}$ & $54,8 \mathrm{~B}$ & $63,4 \mathrm{~B}$ \\
\hline \multirow[t]{3}{*}{$\mathrm{CV}(\%)$} & 7,0 & 5,8 & 5,3 & 4,5 & 4,5 & 4,7 & 7,3 \\
\hline & \multicolumn{7}{|c|}{ Volume de água $(100 \mathrm{ml})$} \\
\hline & \multicolumn{7}{|c|}{ Período de embebição (Horas) } \\
\hline Lotes & 2 & 4 & 6 & 8 & 24 & 48 & 72 \\
\hline 1 & $17,6 \mathrm{~A}$ & $20,0 \mathrm{~A}$ & $22,0 \mathrm{~A}$ & $23,9 \mathrm{~A}$ & $38,5 \mathrm{~A}$ & $57,3 \mathrm{~A}$ & $80,4 \mathrm{~A}$ \\
\hline 2 & $17,4 \mathrm{~A}$ & $19,1 \mathrm{AB}$ & $20,2 \mathrm{AB}$ & $21,1 \mathrm{ABC}$ & $30,2 \mathrm{~B}$ & $42,5 \mathrm{~B}$ & $48,8 \mathrm{~B}$ \\
\hline 3 & $17,0 \mathrm{AB}$ & $19,1 \mathrm{AB}$ & $20,1 \mathrm{AB}$ & $21,0 \mathrm{ABC}$ & $31,8 \mathrm{~B}$ & $43,7 \mathrm{~B}$ & $50,0 \mathrm{~B}$ \\
\hline 4 & $14,5 \mathrm{AB}$ & $17,2 \mathrm{AB}$ & $18,5 \mathrm{AB}$ & $20,0 \mathrm{BC}$ & $30,5 \mathrm{~B}$ & $43,6 \mathrm{~B}$ & $53,3 \mathrm{~B}$ \\
\hline 5 & $17,1 \mathrm{AB}$ & $20,6 \mathrm{~A}$ & $21,5 \mathrm{~A}$ & $22,9 \mathrm{AB}$ & $35,1 \mathrm{AB}$ & $45,4 \mathrm{~B}$ & $53,9 \mathrm{~B}$ \\
\hline 6 & $13,7 \mathrm{~B}$ & $15,9 \mathrm{~B}$ & $17,3 \mathrm{~B}$ & $18,5 \mathrm{C}$ & $31,7 \mathrm{~B}$ & $38,5 \mathrm{~B}$ & $46,3 \mathrm{~B}$ \\
\hline 7 & $16,9 \mathrm{AB}$ & $18,7 \mathrm{AB}$ & $20,6 \mathrm{AB}$ & $22,3 \mathrm{ABC}$ & $34,2 \mathrm{AB}$ & $44,4 \mathrm{~B}$ & $52,8 \mathrm{~B}$ \\
\hline $\mathrm{CV}(\%)$ & 9,3 & 8,2 & 8,2 & 7,8 & 7,7 & 7,0 & 6,7 \\
\hline
\end{tabular}

Médias seguidas da mesma letra, na coluna, não diferem pelo teste de Tukey a 5\% de probabilidade.

No teste de envelhecimento acelerado, conduzido a $45^{\circ} \mathrm{C}$ (Tabela 3), observou-se, em todos os períodos testados, valores de germinação, após envelhecimento inferiores aos obtidos a $41^{\circ} \mathrm{C}$, indicando que aquela temperatura foi mais drástica para as sementes, conforme já se esperava. $\mathrm{O}$ aumento da temperatura é um dos fatores que contribui para acelerar o processo de deterioração das sementes, conforme o princípio básico deste teste (Marcos Filho, 1999). Verificase que o lote 2, considerado nos demais testes de vigor (Tabela 1) e no teste de envelhecimento acelerado a $41^{\circ} \mathrm{C}$ (Tabela 3) como de alto vigor, no teste conduzido a $45^{\circ} \mathrm{C}$, foi classificado entre os de pior desempenho, em todos os períodos de envelhecimento (Tabela 3). Portanto, o envelhecimento acelerado a $45^{\circ} \mathrm{C}$ provocou deterioração excessiva das sementes, dificultando identificar o real nível de vigor dos lotes, especialmente do lote 2. Nota-se que, nos períodos de 48 e 72 horas, apenas o lote 3 foi classificado como de alta qualidade, ficando os lotes 1 e 2, também pertencentes a essa categoria pelos resultados dos testes de frio e emergência de plântulas, como de qualidade inferior.
Santos et al. (2002), recomendaram para sementes de milhodoce o uso da temperatura $42{ }^{\circ} \mathrm{C}$ por $72 \mathrm{~h}$, condições bem próximas às recomendadas no presente trabalho para sementes de mamona.

Observa-se ainda que, no período de 96 horas, a $45^{\circ} \mathrm{C}$, destacaram-se como superiores aos demais, os lotes 1,3 e 7 , sendo os valores absolutos obtidos para os lotes de menor vigor considerados baixos, variando de $26 \%$ a $34 \%$ de germinação, após o envelhecimento (Tabela 3). Esses valores indicam que o processo de deterioração dessas sementes foi bastante acelerado, decorrente das condições a que foram expostas $\left(45^{\circ} \mathrm{C}\right.$ e $100 \%$ UR). É importante ressaltar que, nessa condição, houve elevada contaminação por microrganismos, dificultando as avaliações.

Os lotes de sementes de mamona apresentaram respostas diferenciadas ao estresse imposto pelas combinações de temperatura e período de exposição no envelhecimento acelerado, o que também foi constatado em trabalhos realizados com outras espécies como milho 
(Fessel et al., 2000), aveia-branca (Borsato et al., 2000), sorgo (Miranda et al., 2001) e soja (Lopes et al., 2002). Em geral, as temperaturas de 41 ou $42^{\circ} \mathrm{C}$ por períodos de tempo variando entre 48 e $72 \mathrm{~h}$ foram as condições mais indicadas para a condução do teste de envelhecimento acelerado em sementes dessas espécies, concordando com os resultados obtidos com as sementes de mamona neste trabalho.

Tabela 3 - Germinação (\%) de sementes de mamona submetidas ao teste de envelhecimento acelerado, usandose duas temperaturas e três períodos de exposição.

\begin{tabular}{cccc}
\hline & \multicolumn{3}{c}{$41^{\circ} \mathrm{C}$} \\
\cline { 2 - 4 } Lote & $48 \mathrm{~h}$ & $72 \mathrm{~h}$ & $96 \mathrm{~h}$ \\
\hline & $\ldots \ldots \ldots \ldots \ldots \ldots \ldots \ldots \ldots \ldots \ldots \ldots \ldots \ldots \ldots \ldots \ldots \ldots \ldots \ldots \ldots$ \\
2 & $70 \mathrm{C}$ & $82 \mathrm{~A}$ & $70 \mathrm{AB}$ \\
3 & $82 \mathrm{~A}$ & $78 \mathrm{~A}$ & $86 \mathrm{~A}$ \\
4 & $81 \mathrm{~A}$ & $83 \mathrm{~A}$ & $85 \mathrm{~A}$ \\
5 & $71 \mathrm{C}$ & $66 \mathrm{BC}$ & $57 \mathrm{BC}$ \\
6 & $73 \mathrm{BC}$ & $69 \mathrm{~B}$ & $44 \mathrm{C}$ \\
7 & $56 \mathrm{D}$ & $54 \mathrm{C}$ & $25 \mathrm{D}$ \\
\hline $\mathrm{CV}(\%)$ & $69 \mathrm{C}$ & $67 \mathrm{~B}$ & $63 \mathrm{~B}$ \\
\hline & 5,2 & 7,5 & 11,8 \\
1 & & $45^{\circ} \mathrm{C}$ & \\
\hline 2 & $51 \mathrm{~B}$ & $65 \mathrm{BC}$ & $73 \mathrm{~A}$ \\
3 & $37 \mathrm{C}$ & $41 \mathrm{D}$ & $34 \mathrm{~B}$ \\
4 & $85 \mathrm{~A}$ & $85 \mathrm{~A}$ & $75 \mathrm{~A}$ \\
5 & $56 \mathrm{~B}$ & $51 \mathrm{CD}$ & $26 \mathrm{~B}$ \\
6 & $60 \mathrm{~B}$ & $46 \mathrm{D}$ & $33 \mathrm{~B}$ \\
7 & $50 \mathrm{~B}$ & $41 \mathrm{D}$ & $28 \mathrm{~B}$ \\
\hline $\mathrm{CV}(\%)$ & $60 \mathrm{~B}$ & $76 \mathrm{AB}$ & $77 \mathrm{~A}$ \\
\hline
\end{tabular}

Médias seguidas da mesma letra, na coluna, não diferem pelo teste de Tukey a $5 \%$ de probabilidade.

\section{CONCLUSÕES}

Os testes de frio e de envelhecimento acelerado, a $41^{\circ} \mathrm{C}$ e $100 \%$ UR por 72 horas, são eficientes na identificação do nível de vigor dos lotes de mamona, possibilitando classificação de lotes semelhante àqueles que se obtêm no teste de emergência de plântulas em solo.

$\mathrm{O}$ teste de envelhecimento acelerado a $45^{\circ} \mathrm{C}$ e $100 \%$ UR provoca deterioração excessiva das sementes, dificultando identificar o real nível de vigor dos lotes.

O teste de condutividade elétrica não é adequado para a avaliação do potencial fisiológico das sementes de mamona.

\section{REFERÊNCIAS BIBLIOGRÁFICAS}

ALBUQUERQUE, M.C.F.E.; MORO, F.V.; FAGIOLI, M.; RIBEIRO, M.C. Teste de condutividade elétrica e lixiviação de potássio na avaliação de sementes de girassol. Revista Brasileira de Sementes, Brasília, v.23, n.1, p.1-8, 2001.

ASSOCIATION OF OFFICIAL SEED ANALYSTS. Seed vigour testing handbook. East Lasing, 1983. 88p. (Handbook on Seed Testing. Contribution, 32).

BORSATO, A.V.; BARROS, A.S.R.; AHRENS, D.C.; DIAS, M.C.L.L. Avaliação de testes de vigor para sementes de aveia-branca (Avena sativa $\mathrm{L}$.). Revista Brasileira de Sementes, Brasília, v.22, n.1, p.163-168, 2000 .

BRASIL. Ministério da Agricultura e Reforma Agrária. Regras para análise de sementes. Brasília: CLAV/ DNDV/SNDA/MA, 1992. 365p.

DEPARTAMENTO FEDERAL DEAGRICULTURA DE

SÃO PAULO. Disponível em: <http://www.dfasp.gov.br// isefag_vegetal/doc/legislacao/ lanexo_vi_da_instrucao_normativa_25 de $16-12$ J 2005.pdf $>$. Acesso em: 28 ago. 2007.

DIAS, D.C.F.S.; MARCOS FILHO, J. Teste de condutividade elétrica para avaliação do vigor de sementes de soja. Scientia Agricola, Piracicaba, v.53, n.1, p.31- 42, 1996.

FESSEL, S.A.; RODRIGUES, T.J.D.; FAGIOLI, M.; VIEIRA, R.D. Temperatura e período de exposição no teste de envelhecimento acelerado em sementes de milho. Revista Brasileira de Sementes, Brasília, v.22, n.2, p.163-170, 2000.

FONSECA, N.R.; MYCZKOWSKI, M.L.; PRIOR, M.; SÁ, R.O.; NAKAGAWA, J.; CAVARIANI, C.;

ZANOTTO, M.D. Testes de avaliação da viabilidade e do vigor em sementes de mamona. In: CONGRESSO BRASILEIRO DE MAMONA - ENERGIA E SUSTENTABILIDADE, 1., 2004, Campina Grande. Anais... Campina Grande: Embrapa Algodão, 2004. p.712.

FREIRE, R.M.M. Ricinoquímica. In: AZEVEDO, D.M.P.; LIMA, E.F. (Eds.). $O$ agronegócio da mamona no Brasil. Brasília: Embrapa Informação Tecnológica, 2001. p.295-335. 
GASPAR, C.M.; NAKAGAWA, J. Teste de condutividade elétrica em função do número de sementes e da quantidade de água para sementes de milheto. Revista Brasileira de Sementes, Curitiba, v.24, n.2, p.70-76, 2002.

GUEDES, R. S.; ALVES, E. U.; GONÇALVES, E. P.; SANTOS, S. do R. N. dos; LIMA, C. R. de. Testes de vigor na avaliação da qualidade fisiológica de sementes Erythrina velutina Willd. (Fabaceae-Papilionoideae). Ciência e Agrotecnologia, Lavras, v. 33, n. 5, p. 13601365, set./out., 2009.

HAMPTON, J.G.; TEKRONY, D.M. Handbook of vigour test methods. Zurich: ISTA, 1995. 117p.

INTERNATIONAL SEED TESTING ASSOCIATION. Handbook of vigour test methods. 3.ed. Zurich, 1995. 117p.

LOEFFLER, N.L.; MEIER, J.L.; BURRIS, J.S.

Comparasion of two cold test procedures for use in maize drying studies. Seed Science and Technology, Zurich, v.13, n.3, p.653-658, 1985.

LOEFFLER, T.M.; TEKRONY, D.M.; EGLI, D.B. The bulk conductivity test as an indicator of soybean seed quality. Journal of Seed Technology, Zurich, v.12, n.1, p.37-53, 1988.

LOPES, J.C.; MARTINS-FILHO, S.; TAGLIAFERRE, C.; RANGEL, O.J.P. Avaliação da qualidade fisiológica de sementes de soja produzidas em Alegres-ES. Revista Brasileira de Sementes, Brasília, v.24, n.1, p.51-58, 2002.

MARCOS FILHO, J. Teste de envelhecimento acelerado. In: KRZYZANOWSKI, F.C.; VIEIRA, R.D.; FRANÇANETO, J.B. (Eds.). Vigor de sementes: conceitos e testes. Londrina: Abrates, 1999. cap.3, p.1-24.

MIRANDA, D.M.; NOVEMBRE, A.D.L.C.; CHAMMA, H.M.C.P. Avaliação do potencial fisiológico de sementes de sorgo pelo teste de envelhecimento acelerado.

Revista Brasileira de Sementes, Brasília, v.23, n.1, p.226-231, 2001.

NAKAGAWA, J. Testes de vigor baseados no desempenho das plântulas. In: KRZYZANOWSKI, F.C.; VIEIRA, R.D.; FRANÇA-NETO, J.B. (Eds.). Vigor de sementes: conceitos e testes. Londrina: Abrates, 1999. cap.2, p.1-24.
PARRELLA, N.N.L.D.; CARVALHO, M.L.M.; PARRELLA, R.A.C.; FIDANZA, L. Qualidade de sementes de mamona (Ricinus communis L.) utilizadas no Estado de Minas Gerais. In: CONGRESSO BRASILEIRO DE PLANTAS OLEAGINOSAS, ÓLEOS, GORDURAS E BIODIESEL, 4., 2007, Varginha. Anais... Lavras: UFLA, 2007. p.906-913.

ROCHA, M.S.; BRAGA JÚNIOR, J.M.; BRUNO, R.L.A.; VIANA, J.S.; MOURA, M.F.; BELTRÃO, N.E.M.; GUEDES, R.S. Teste de envelhecimento acelerado em sementes de mamona cultivar BRS Energia. In: CONGRESSO BRASILEIRO DE PLANTAS OLEAGINOSAS, ÓLEOS, GORDURAS E BIODIESEL, 4., 2007, Varginha. Anais... Lavras: UFLA, 2007. p.1421-1431.

SANTOS, P.M.; GONDIM, T.C.O.; ARAÚJO, E.F.; DIAS, D.C.F.S. Avaliação da qualidade fisiológica de sementes de milho-doce pelo teste de envelhecimento acelerado. Revista Brasileira de Sementes, Pelotas, v.24, n.1, p.91-96, 2002.

SAS INSTITUTE. SAS/STAT user's guide. Version 6.4 ed. Cary, 1989.

SAVY FILHO, A. Mamona tecnologia agrícola. Campinas: EMOPI, 2005. 105p.

SOUZA, L.A.; CARVALHO, M.L.M.; KATAOKA, V.Y.; COELHO, D.S.; SANTOS-NETO, A.L. Teste de condutividade elétrica para avaliação da qualidade de sementes de mamona. In: CONGRESSO BRASILEIRO DE PLANTAS OLEAGINOSAS, ÓLEOS, GORDURAS E BIODIESEL, 4., 2007, Varginha. Anais... Lavras: UFLA, 2007. p.409-418.

TAO, J.K. Factors causing variations in the conductivity test for soybean seeds. Journal of Seed Technology, Zurich, v.3, n.1, p.10-18, 1978.

VANZOLINI, S.; NAKAGAWA, J. Teste de condutividade elétrica em sementes de amendoim: efeitos de temperatura e de período de embebição. Revista Brasileira de Sementes, Brasília, v.21, n.1, p.4145, 1999.

VIEIRA, R.D.; KRZYZANOWSKI, F.C. Teste de condutividade elétrica. In: KRZYZANOWSKI, F.C.; VIEIRA, R.D.; FRANÇA-NETO, J.B. (Eds.). Vigor de sementes: conceitos e testes. Londrina: Abrates, 1999. cap.4, p.1-20. 